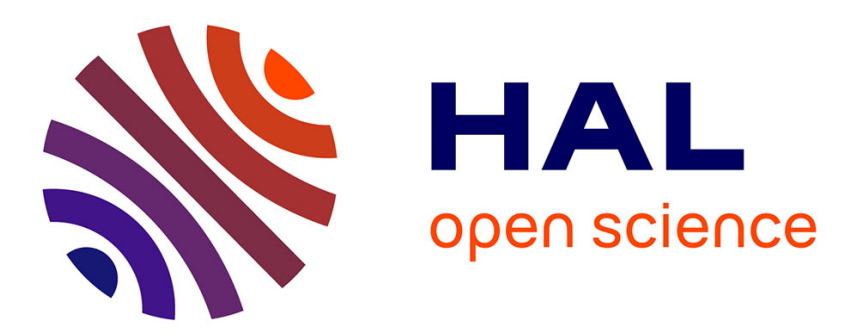

\title{
Panel measurement method for the determination of scattering coefficients and edge diffraction in an open water tank
}

Laetitia Roux, Monique Pouille, Christian Audoly, Anne-Christine Hladky

\section{- To cite this version:}

Laetitia Roux, Monique Pouille, Christian Audoly, Anne-Christine Hladky. Panel measurement method for the determination of scattering coefficients and edge diffraction in an open water tank. Journal of the Acoustical Society of America, 2020, 10.1121/10.0000727 . hal-03019826

\section{HAL Id: hal-03019826 \\ https://hal.science/hal-03019826}

Submitted on 23 Nov 2020

HAL is a multi-disciplinary open access archive for the deposit and dissemination of scientific research documents, whether they are published or not. The documents may come from teaching and research institutions in France or abroad, or from public or private research centers.
L'archive ouverte pluridisciplinaire HAL, est destinée au dépôt et à la diffusion de documents scientifiques de niveau recherche, publiés ou non, émanant des établissements d'enseignement et de recherche français ou étrangers, des laboratoires publics ou privés. 


\title{
Panel measurement method for the determination of scattering coefficients and edge diffraction in an open water tank
}

Laetitia Roux, ${ }^{1, a)}$ Monique Pouille, ${ }^{1}$ Christian Audoly, ${ }^{2}$ and Anne-Christine Hladky ${ }^{1}$

${ }^{1}$ Centre National de la Recherche Scientifique, Centrale Lille, ISEN, Univ. Lille, Univ. Valenciennes, UMR 8520-IEMN,

41 Boulevard Vauban, 59800 Lille, France

${ }^{2}$ Naval Group Research, 199 Avenue Pierre-Gilles de Gennes, 83190 Ollioules, France

\begin{abstract}
:
Material effective properties of composite media or their acoustic performance are often required to be experimentally determined or verified. For underwater applications, systems can be characterized in an open water tank in which a conventional measurement configuration consists of the panel, an acoustic source, and two hydrophones, one on either side of the panel. However, panel measurements may be greatly complicated by waves diffracted by the panel edges. The three-point method is a technique that decomposes total pressure into contributions of the incident, reflected, transmitted, and edge-diffracted pressures. These contributions are determined using measurements at three positions. Reflection and transmission coefficients can then be obtained by removing the contribution of the edge-diffracted waves. The three-point method is applied to an aluminum reference panel for which the experimental scattering coefficients are in very good agreement with those of an infinite panel. The validity limits of the method are also studied. Finally, a pressure mapping shows that the three-point method leads to an accurate determination of the reflection and transmission coefficients for different positions despite strong variations of the edge-diffracted pressure. (c) 2020 Acoustical Society of America. https://doi.org/10.1121/10.0000727

(Received 7 November 2019; revised 30 December 2019; accepted 28 January 2020; published online 14 February 2020)

[Editor: Stephen Paul Robinson]

Pages: 1104-1112
\end{abstract}

\section{INTRODUCTION}

Composite materials are commonly used for a range of underwater applications such as acoustic windows, acoustic barriers, or acoustic coatings applied to the outer surface of submerged vessels. They generally consist of elastomeric materials based on natural rubber or polyurethane and may include micro-inclusions or micro-voids to modulate the effective speed of sound in the medium. They may also contain larger inclusions designed to resonate at specific frequencies, based on the concept of metamaterials. ${ }^{1}$ Acoustic performance of such coatings can be predicted using analytical models ${ }^{2}$ or finite element simulations. ${ }^{3,4}$ However, these approaches require an accurate knowledge of the acoustic properties of the elastomeric matrix and/or the composite coating, which can be obtained experimentally. In any case, a panel must be characterized to verify its performance, commonly expressed in terms of reflection and transmission coefficients from which other coefficients may be derived. ${ }^{5}$

Experimental characterization of a panel requires the insonification of the sample by a known acoustic field. The reflection loss of a sample is obtained from the acoustic field reflected by the sample, while the transmission loss is calculated with the acoustic field passing through the sample. ${ }^{6}$ The reflection and transmission coefficients can be measured in an impedance tube, a pressurized water tank, or an open water tank. ${ }^{7}$ This paper focuses on normal incidence characterization techniques for panels in open water tanks.

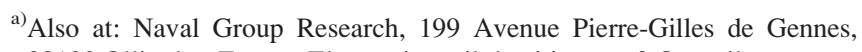
83190 Ollioules, France. Electronic mail: laetitia.roux.fr@gmail.com
One of the main difficulties of experimental measurements is the reproduction of the set of assumptions used for the theoretical or numerical calculations, which are difficult to implement in practice. For example, theoretical models or finite element calculations on periodic systems often consider infinite lateral dimensions, which is obviously impossible to achieve, and making an infinite panel assumption becomes problematic for lowfrequency measurements. One of the main parasitic effects resulting from the finite lateral dimensions of the panel is the diffraction of acoustic waves by its edges. There are techniques ${ }^{8}$ and models ${ }^{9,10}$ to obtain direct evidence of the edge-diffraction phenomenon. It has even been shown that the importance of the edge diffraction perturbations is largely influenced by the material properties of the sample. ${ }^{11}$ Edge diffraction is particularly complicating measurements for panels made of acoustically soft materials, since the amplitude of the transmitted waves can be very small compared to that of the diffracted waves. Moreover, if the speed of sound in the sample is rather low, the diffracted waves can overlap the reflected and transmitted signals, which makes the differentiation difficult. ${ }^{12,13}$ Edge diffraction also highly contaminates measurements on acoustically hard panels for which the diffracted pattern shows strong variations in space within measurement planes. ${ }^{9}$

Several methods have been developed to estimate the amplitude of diffracted waves. One of them requires an air box to be placed in front of the panel for which the transmission loss is more than $60 \mathrm{~dB}$. Thus, the signal received on the transmitted side is assumed to result from the sole contribution of the edge-diffracted waves. ${ }^{14}$ An alternative technique has recently been presented which involves surrounding the 
panel in a reflective baffle so that its edges are less excited, thus reducing the contamination. ${ }^{15}$ Another experimental mean for reducing the corruptive effects of edge diffraction is the use of a parametric array as the insonification is maximized at the center of the panel and reduced at the edges. ${ }^{16}$ The directivity of a parametric array for low-frequency waves is obtained by the interaction of high-frequency waves, which implies a nonlinear mixing of the sound. ${ }^{17}$ Nevertheless, it must be ensured that nonlinear effects do not complicate or invalidate the measurement process, ${ }^{18}$ so a low-pass acoustic filter is required in the measurement zone to attenuate the higher frequency primary waves. A surface receiver may also be used to reduce the scattered field perturbations on the transmission coefficient measurements. This approach helps reduce the effects of the edge-diffracted pressure, assuming that the optimal size and position of the receiver have been found. ${ }^{10}$ Perturbations caused by edge-diffracted waves can also be minimized during the post-processing by considering the transient-state reflected and transmitted signals, which are less contaminated. The transient-state signals are then extrapolated to obtain the steady-states used to derive the scattering coefficients. This process, known as the ONION method, has given good results for thick multilayered panels made of materials whose properties are known. ${ }^{13,19,20}$ Therefore, there are techniques that have been implemented to reduce the edge diffraction effects, but they all have their own limitations as they often require the manufacture or the use of some specific equipment, or they may impose constraints on the panel design (thickness, material, etc.). Moreover, even with these techniques, a good compromise must still be found for the position of the hydrophones with respect to the panel. They must be positioned close enough to reduce the detection of diffracted waves and far enough to avoid the overlap of the incident and reflected signals.

In this paper, a method is presented for acoustic panel measurements in open water tanks. This method is based on a conventional measurement configuration consisting of the panel, an acoustic source, and two hydrophones, one on either side of the panel. In the experimental setup, only three different positions for the hydrophones are considered. This method, called the three-point method, provides an estimation of the amplitude of the edge-diffracted waves as well as the amplitudes of the transmitted, reflected, and incident waves. This paper presents this method and aims to assess its robustness, advantages, and limitations. For this purpose, several experiments are conducted in a water tank on an aluminum reference panel. The method is first validated by comparing the experimental coefficients to theoretical predictions for a panel assumed to be of infinite lateral dimensions. Then, limitations of the three-point method are presented through three tests. Finally, a pressure mapping is realized for the aluminum panel to illustrate its diffraction pattern.

\section{THREE-POINT METHOD}

Panels are characterized for a quasi-plane incident wave-front at normal incidence. The phase convention is $e^{\mathrm{i}(\omega t-k x)}$, where $\mathrm{i}=\sqrt{-1}, \omega$ is the radian frequency, $k$ is the longitudinal wavenumber, $t$ denotes time, and $x$ defines the position on the longitudinal axis. One hydrophone is placed in front of the panel at position $A$ to measure the sum of the reflected pressure $p_{r}$ and the incident pressure $p_{i}$. Another hydrophone is located at position $B$ behind the panel to obtain the transmitted pressure $p_{t}$, as shown in Fig. 1 .

These pressures are used to calculate the reflection and transmission coefficients of a panel at positions $A$ and $B$, as per Eqs. (1) and (2). A phase shift is then applied to these equations so that $R$ corresponds to the reflection coefficient at the front face of the panel and $T$ corresponds to the transmission coefficient between the front and back faces,

$$
\begin{aligned}
& R(A)=\frac{p_{r}(A)}{p_{i}(A)}, \\
& T(B)=\frac{p_{t}(B)}{p_{i}(A)} .
\end{aligned}
$$

However, these pressures are contaminated with the contribution of the edge-diffracted waves. The three-point method provides a way to extract these specific pressure contributions. The method is detailed below, using Fig. 2 to illustrate the experimental setup specific to the method.

On both sides of the panel, total acoustic pressure is measured at three points. The three-point method then treats the reflection and transmission sides in slightly different ways. For the determination of the reflection coefficient, the three points $A_{1}, A_{2}$, and $A_{3}$ are considered. The central position $A_{2}$ is taken as a reference with the distances $d_{A 12}=A_{1} A_{2}$ and $d_{A 23}=A_{2} A_{3}$. At each point, the total pressure $p$ can be expressed as a function of the contributions of the incident pressure $p_{i}$ and the reflected pressure $p_{r}$ at the central position, as well as the diffracted pressure on the reflection side $p_{\text {diff }_{R}}$,

$$
\left\{\begin{array}{l}
p\left(A_{1}\right)=p_{i}\left(A_{2}\right) e^{-\mathrm{i} k d_{A 12}}+p_{r}\left(A_{2}\right) e^{\mathrm{i} k d_{A 12}}+p_{\operatorname{diff}_{R}}\left(A_{1}\right) \\
p\left(A_{2}\right)=p_{i}\left(A_{2}\right)+p_{r}\left(A_{2}\right)+p_{\operatorname{diff}_{R}}\left(A_{2}\right) \\
p\left(A_{3}\right)=p_{i}\left(A_{2}\right) e^{\mathrm{i} k d_{A 23}}+p_{r}\left(A_{2}\right) e^{-\mathrm{i} k d_{A 23}}+p_{\operatorname{diff}_{R}}\left(A_{3}\right)
\end{array}\right.
$$

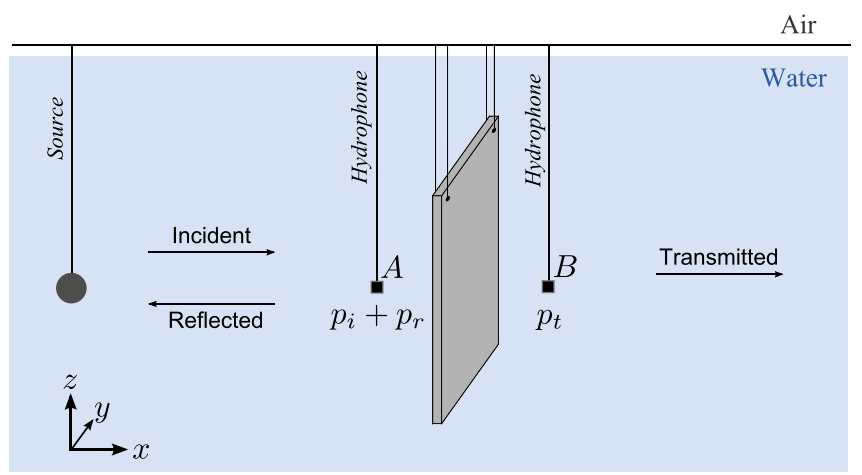

FIG. 1. (Color online) Conventional setup for panel measurements in an open water tank. 


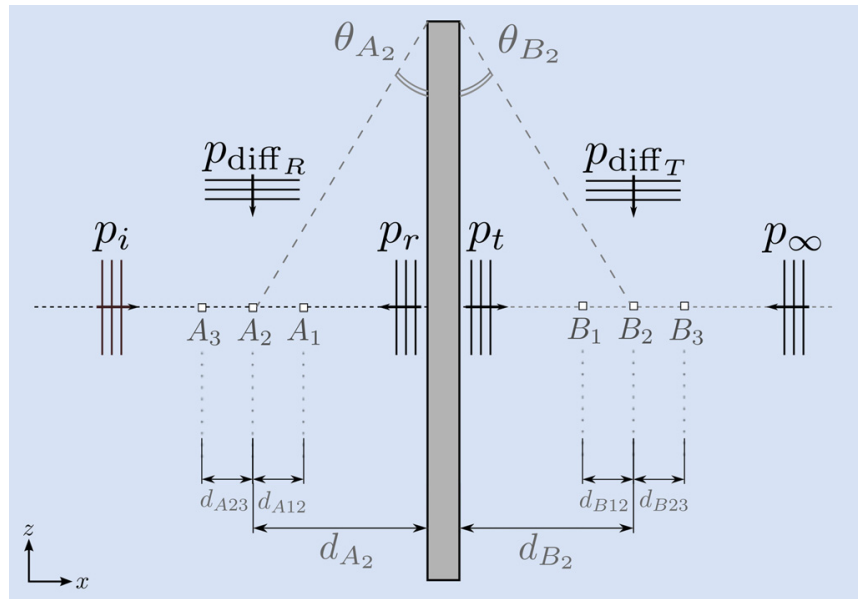

FIG. 2. (Color online) Experimental setup specific to the application of the three-point method. Points $A_{1}, A_{2}$, and $A_{3}$ correspond to the three positions taken by the hydrophone on the reflection side, while points $B_{1}, B_{2}$, and $B_{3}$ are the positions of the hydrophone on the transmission side. Pressure contributions and geometric parameters are also presented.

It is then assumed that the total contribution of the edge-diffracted pressure is equal in these three positions when the three points are placed close to each other on the $x$ axis. To legitimize this hypothesis, the analytical model presented in the Appendix is used by comparing the edgediffracted pressures in different positions. This hypothesis thus leads to

$$
p_{\text {diff }_{R}}\left(A_{1}\right)=p_{\text {diff }_{R}}\left(A_{2}\right)=p_{\text {diff }_{R}}\left(A_{3}\right)=p_{\text {diff }_{R}} .
$$

System (3) can be simplified and written into a matrix form, which gives

$$
\left(\begin{array}{l}
p\left(A_{1}\right) \\
p\left(A_{2}\right) \\
p\left(A_{3}\right)
\end{array}\right)=\left[\begin{array}{ccc}
e^{-\mathrm{i} k d_{A 12}} & e^{\mathrm{i} k d_{A 12}} & 1 \\
1 & 1 & 1 \\
e^{\mathrm{i} k d_{A 23}} & e^{-\mathrm{i} k d_{A 23}} & 1
\end{array}\right]\left(\begin{array}{c}
p_{i}\left(A_{2}\right) \\
p_{r}\left(A_{2}\right) \\
p_{\mathrm{diff}_{R}}
\end{array}\right) .
$$

By inverting Eq. (5), the pressures $p_{i}\left(A_{2}\right), p_{r}\left(A_{2}\right)$, and $p_{\text {diff }_{R}}$ are obtained,

$$
\left(\begin{array}{c}
p_{i}\left(A_{2}\right) \\
p_{r}\left(A_{2}\right) \\
p_{\mathrm{diff}}
\end{array}\right)=\left[\begin{array}{ccc}
e^{-\mathrm{i} k d_{A 12}} & e^{\mathrm{i} k d_{A 12}} & 1 \\
1 & 1 & 1 \\
e^{\mathrm{i} k d_{A 23}} & e^{-\mathrm{i} k d_{A 23}} & 1
\end{array}\right]^{-1}\left(\begin{array}{c}
p\left(A_{1}\right) \\
p\left(A_{2}\right) \\
p\left(A_{3}\right)
\end{array}\right) .
$$

Once the total pressures at points $A_{1}, A_{2}$, and $A_{3}$ are measured, the contribution of the incident, reflected, and edge-diffracted pressures can be deduced using the previous system. Reflection coefficient at point $A_{2}$ is then obtained using Eq. (1).

On the transmission side, the same approach is adopted, considering the three points $B_{1}, B_{2}$, and $B_{3}$ and the distances $d_{B 12}=B_{1} B_{2}$ and $d_{B 23}=B_{2} B_{3}$, where position $B_{2}$ is taken as the reference. In this case, the pressure field is decomposed into contributions of the transmitted pressure $p_{t}$, the diffracted pressure $p_{\operatorname{diff}_{T}}$, as well as the pressure $p_{\infty}$, known as infinite pressure. The latter describes a wave coming from infinity in the direction of decreasing $x$. On the reflection side, this pressure would correspond to the contribution of reflected waves, but on the transmission side, only waves reflected on the water tank wall could be travelling in this direction. Nevertheless, such waves are not recorded within the acquisition time window considered for the measurements. Thus, the infinite pressure is theoretically supposed to be zero. The case of $p_{\infty}$ being significantly non-null would then be attributed to other distortions between the model and experimental setup. On the transmission side, the same hypothesis on the edgediffracted pressure is used, so $p_{\text {diff }}$ is also assumed to be equal for each of the three points. The total pressures at points $B_{1}$, $B_{2}$, and $B_{3}$ are therefore expressed by

$$
\left\{\begin{array}{l}
p\left(B_{1}\right)=p_{t}\left(B_{2}\right) e^{\mathrm{i} k d_{B 12}}+p_{\infty}\left(B_{2}\right) e^{-\mathrm{i} k d_{B 12}}+p_{\text {diff }_{T}}, \\
p\left(B_{2}\right)=p_{t}\left(B_{2}\right)+p_{\infty}\left(B_{2}\right)+p_{\mathrm{diff}_{T}}, \\
p\left(B_{3}\right)=p_{t}\left(B_{2}\right) e^{-\mathrm{i} k d_{B 23}}+p_{\infty}\left(B_{2}\right) e^{\mathrm{i} k d_{B 23}}+p_{\text {diff }_{T}} .
\end{array}\right.
$$

The contributions of the transmitted, infinite, and diffracted pressure are then be calculated with

$$
\left(\begin{array}{c}
p_{t}\left(B_{2}\right) \\
p_{\infty}\left(B_{2}\right) \\
p_{\text {diff }}
\end{array}\right)=\left[\begin{array}{ccc}
e^{\mathrm{i} k d_{B 12}} & e^{-\mathrm{i} k d_{B 12}} & 1 \\
1 & 1 & 1 \\
e^{-\mathrm{i} k d_{B 23}} & e^{\mathrm{i} k d_{B 23}} & 1
\end{array}\right]^{-1}\left(\begin{array}{c}
p\left(B_{1}\right) \\
p\left(B_{2}\right) \\
p\left(B_{3}\right)
\end{array}\right) .
$$

With the measurements of the total pressure at points $B_{1}, B_{2}$, and $B_{3}$, the contributions of the transmitted, infinite, and edge-diffracted pressures can be deduced using the previous system. The transmission coefficient between $A_{2}$ and $B_{2}$ is then obtained using Eq. (2).

\section{APPLICATION}

\section{A. Experimental setup}

Measurements are conducted in a large open water tank, shown in Fig. 3, which is $8 \mathrm{~m}$ long, $6 \mathrm{~m}$ wide, and $7 \mathrm{~m}$ deep. This facility is located in the academic laboratory ISEN in Lille, France. The experimental setup consists of two hydrophones placed on either side of the panel. The position of the receivers on the $x$ axis and $y$ axis can be easily modified using Rexroth motors (Bosch Rexroth, Lohr am Main, Germany), which are able to move the hydrophones with an accuracy to the order of one-tenth of a millimeter. The experimental bench is fully controlled via a LabView interface (National Instruments, Austin, Texas), which has been coded in such a way that the user simply needs to indicate the frequency range as well as the different positions of the hydrophones along the $x$ axis. It is also possible to automatically change the hydrophone positions for each frequency, so as to define the distance between two positions in terms of wavelength $\lambda$ instead of absolute distance.

In the water tank, the hydrophones and the projector are completely immersed to a depth of $2.73 \mathrm{~m}$, corresponding to the position of the panel's center. The spherical projector is 


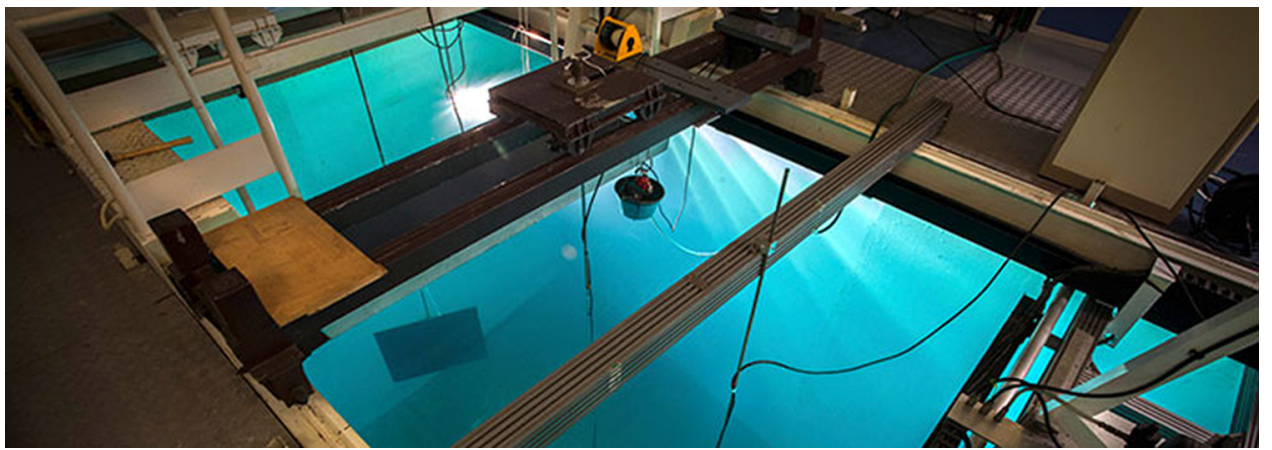

FIG. 3. (Color online) Open Water Tank Facility of ISEN, Lille, France. Jaochin Dassonville.

placed $2.87 \mathrm{~m}$ away from the panel so that the assumption of incident plane waves is made. The water temperature of $15.5^{\circ} \mathrm{C}$ remains stable for all measurements. Under such conditions, the speed of sound in water is estimated at $1489 \mathrm{~m} \mathrm{~s}^{-1}$.

The emitted signal is a burst defined by the frequency of study and a duration time of $2.2 \mathrm{~ms}$. The signal received in water is the signal resulting from the contribution of the incident, reflected, and edge-diffracted waves in front of the panel and from the contribution of the transmitted and edgediffracted waves behind the panel. Useful information can be retrieved from the signals when they reach a steady-state. An acquisition window is thus defined to record these steady-state signals, also preventing signal contamination by the waves reflected on the tank's walls. A preprocessing step is then performed to check the quality of the recorded signals. It detects pairs of positions $X$ and frequencies $f$ for which destructive interference of all the waves, including edge-diffracted waves, makes it impossible to record a steady-state signal, necessary to accurately calculate the scattering coefficients. Such pairs $(X, f)$ resulting in poor quality signals are detected using the frequencies extracted from signals recorded in the acquisition window. When a retrieved frequency is significantly different from the measurement frequency of interest, the pair $(X, f)$ is not taken into account in the post-processing step.

\section{B. Validation}

The three-point-method is applied on a reference panel made of 5083 aluminum alloy, dimensions $1 \mathrm{~m} \times 1 \mathrm{~m}$, and thickness $4 \mathrm{~cm}$. Hydrophone positions are always kept symmetrical about the panel, so the notation $X$ is used independently of the side for the position $X=A$ or $X=B$. Positions for the hydrophones are given in Table I. A distance of $\lambda / 15$ is considered between the hydrophone positions, thus, changing for each frequency within the frequency range $3-13 \mathrm{kHz}$. With this distance of $\lambda / 15$ between the hydrophone positions, the edge-diffracted pressure differs by less than $0.6 \%$ from

TABLE I. Geometric parameters for validation of the three-point method.

\begin{tabular}{lccc}
\hline \hline$d_{X_{2}}(\mathrm{~cm})$ & $d_{X_{1,2}}(\lambda)$ & $d_{X_{2,3}}(\lambda)$ & $\theta\left(^{\circ}\right)$ \\
\hline 8 & $\lambda / 15$ & $\lambda / 15$ & 9.1 \\
\hline \hline
\end{tabular}

J. Acoust. Soc. Am. 147 (2), February 2020 one position to another, according to the analytical model in the Appendix. The configuration defined in Table I therefore complies with the hypothesis that the edge-diffracted pressure is equal in the three measurement positions, as per Eq. (4). Moreover, with this distance, the total pressures at the three positions are sufficiently different [cf. test 3 in Sec. IIIC (Validity limits)].

The total pressures recorded at the three positions on each side of the panel are plotted in Fig. 4. As previously mentioned, the total pressure on the reflection side may reach local minima, resulting from destructive interference. Pairs of positions/frequencies $(X, f)$ that have been removed using the preprocessing step are highlighted with circles. On the reflection side, the total pressures are used to derive the incident pressure $p_{i}$, the reflected pressure $p_{r}$, and the diffracted pressure on the reflection side $p_{\operatorname{diff}_{R}}$. On the transmission side, the three-point method leads to the transmitted pressure $p_{t}$ as well as the diffracted pressure $p_{\text {diff }_{T}}$. Pressure $p_{\infty}$ coming from infinity and travelling toward the negative $x$ is also obtained for the transmission side. As the infinite pressure is supposed to be zero, the validity of the threepoint method can be checked. All these calculated pressures are shown in Fig. 5.

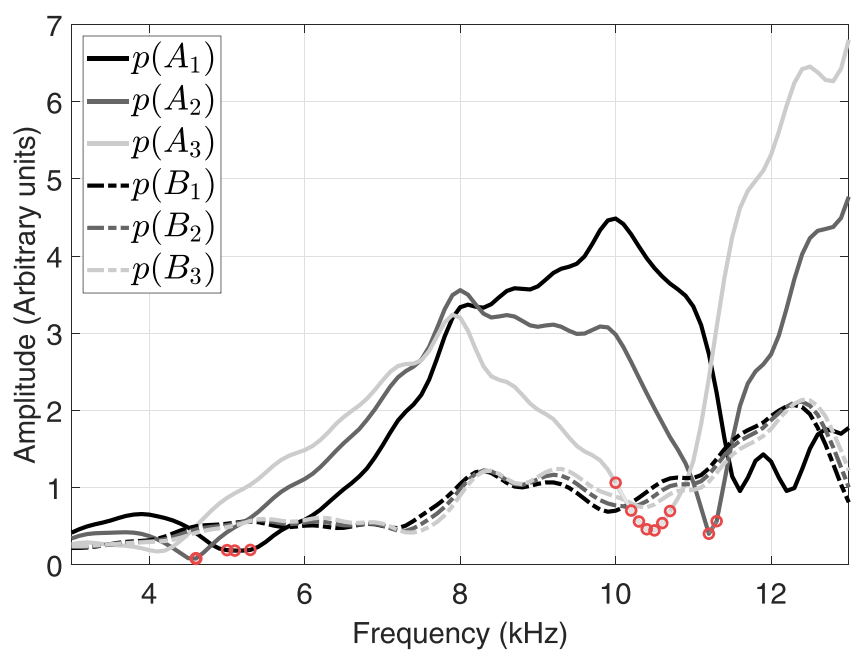

FIG. 4. (Color online) Pressure amplitudes measured at the three positions $A_{1}, A_{2}$, and $A_{3}$ for the reflection side and at the three positions $B_{1}, B_{2}$, and $B_{3}$ for the transmission side. The circles represent pairs $(X, f)$ of destructive interference. 
(a)

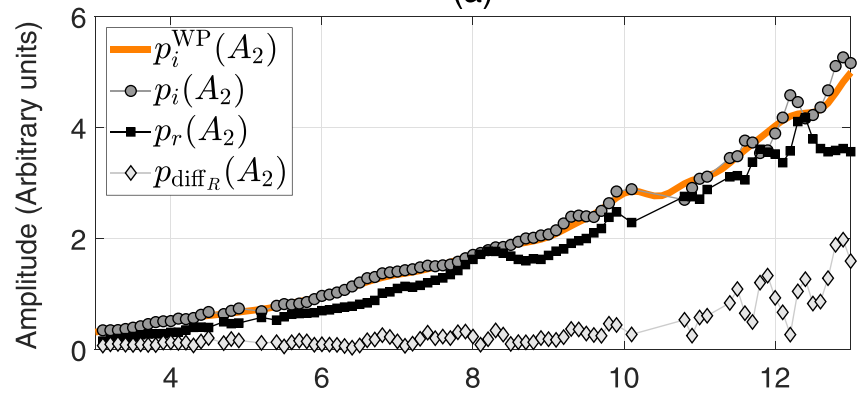

(b)

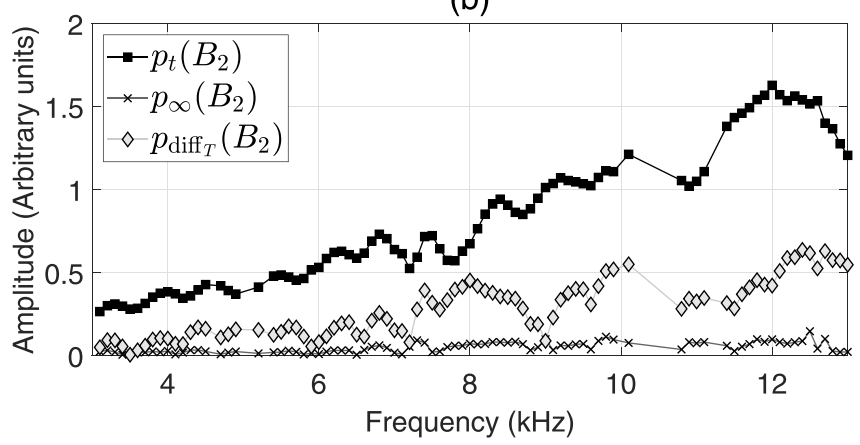

FIG. 5. (Color online) (a) Incident pressure $p_{i}$, reflected pressure $p_{r}$, and diffracted pressure $p_{\text {diff }_{R}}$ at the central position $A_{2}$ on the reflection side. The incident pressure measured without panel $\left(p_{i}^{\mathrm{WP}}\right)$ is also plotted. (b) Transmitted pressure $p_{t}$, infinite pressure $p_{\infty}$, and diffracted pressure $p_{\operatorname{diff}_{T}}$ at the central position $B_{2}$ on the transmission side.

The incident pressure is compared to that obtained by measurements without panel, referred to as $p_{i}^{\mathrm{WP}}$. It can be seen that both are consistent over the whole frequency range, validating the estimation of the incident pressure provided by the three-point method. In addition, the infinite pressure $p_{\infty}$ seems to be almost zero over the entire frequency range, which is expected when the three-point method is valid and correctly implemented. It can also be noted that the diffracted pressure, on both sides, oscillates approximately between $2 \%$ and $70 \%$ of the total pressure, showing that the edgediffraction effects cannot be neglected.

Finally, reflection and transmission coefficients are obtained analytically for a medium of infinite lateral dimensions. ${ }^{21}$ The material properties for aluminum are $2700 \mathrm{~kg} \mathrm{~m}^{-3}$ for the mass density and $6183 \mathrm{~m} \mathrm{~s}^{-1}$ for the longitudinal speed of sound. Since the three-point method eliminates edgediffraction contributions, the experimental coefficients can be directly compared to those of an infinite panel, as in Fig. 6 . Experimental coefficients have also been fitted to a quadratic curve. This interpolation weakens the few slight discrepancies, which are mainly explained by the structural resonant modes of the finite panel, such as the plate bending modes. ${ }^{10}$ Therefore, the three-point method leads to reflection and transmission coefficients that fit well with the ones calculated analytically for an infinite panel. Data recorded at the central position $X_{2}$ are also used to calculate the scattering coefficients as per the direct conventional method, using a single point. There is a closer agreement between the three-point analysis result and theoretical (infinite plate) calculation, than between the conventional (a)

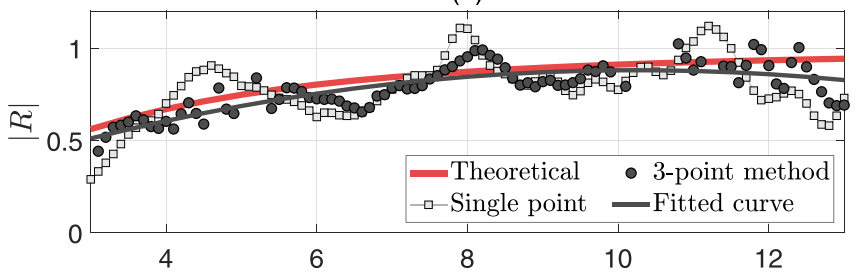

(b)

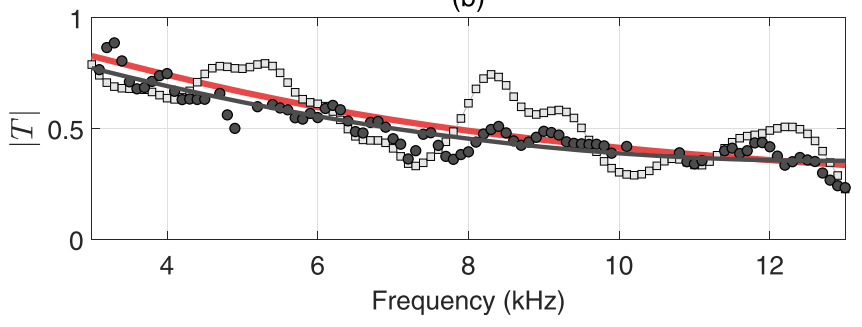

FIG. 6. (Color online) Modulus of (a) the reflection coefficient and (b) the transmission coefficient obtained analytically for a panel of infinite lateral dimensions and measured with the direct and the three-point method. Positions for the three-point method are defined in Table I, and the central position $X_{2}$ is used for the direct single point method. Results from the three-point method are fitted to a quadratic curve.

single point analysis result and theoretical calculation, which highlights the benefit of using the three-point method instead of direct measurements to remove the contribution of the edgediffraction, since its effects are also strongly dependent on the hydrophone position, as shown later in Sec. IV.

\section{Validity limits}

It has been shown with the previous example that when the hypothesis on the diffracted pressure is satisfied, the three-point method leads to reflection and transmission coefficients that fit well with those of an infinite panel. The current section studies the limits of validity of the method based on three tests defined in Table II. Measurements for these tests have been conducted between 1 and $15 \mathrm{kHz}$. The first test considers three points close to each other (separated by $1 \mathrm{~cm}$ so that the hypothesis on the diffracted pressure being equal in the three points is assumed to be satisfied) but far away from the panel, where the central position forms an angle $\theta=25.6^{\circ}$ with the panel face. In the second test, a significantly greater distance between hydrophone positions is considered, with $d_{X_{1,2}}=d_{X_{2,3}}=10 \mathrm{~cm}$, thus challenging the hypothesis on the edge-diffracted pressure equal in the three points. Finally, test 3 considers three positions so that the angle $\theta$ is now equal to $5.7^{\circ}$, where hydrophone positions are separated from each other by only $0.5 \mathrm{~cm}$. Test 3 then satisfies the hypothesis on the edge-diffracted waves.

TABLE II. Configuration parameters tested to assess limitations of the three-point method.

\begin{tabular}{lcccc}
\hline \hline & $d_{X_{2}}(\mathrm{~cm})$ & $d_{X_{1,2}}(\mathrm{~cm})$ & $d_{X_{2,3}}(\mathrm{~cm})$ & $\theta\left({ }^{\circ}\right)$ \\
\hline Test 1 & 24 & 1 & 1 & 25.6 \\
Test 2 & 15 & 10 & 10 & 16.7 \\
Test 3 & 5 & 0.5 & 0.5 & 5.7 \\
\hline \hline
\end{tabular}


For these three tests, the reflection and transmission coefficients given by the three-point method are plotted in Fig. 7. It is first observed that these experimental scattering coefficients are very different from those of an infinite panel obtained theoretically (lines without markers). Moreover, all of them reach values higher than one, which makes no sense physically for a passive panel. Results for each test are further analyzed.

In test 1 , the relative difference between the edgediffracted pressures in each point remains less than $1 \%$ according to the model in the Appendix. Even though the hypothesis on the edge-diffracted pressure can be considered as valid here, the experimental coefficients are very different from the theoretical ones because when the hydrophones are this far away from the panel, the total pressure cannot be decomposed into three distinct contributions as per Fig. 2. It is indeed difficult to distinguish the reflected/transmitted waves from the edge-diffracted waves since they tend to arrive at the same time and incidence angle on the hydrophones. The angle $\theta$ should therefore remain less than $15^{\circ}$.

Test 2 considers a greater distance between hydrophone positions. This configuration gives acceptable results at very low frequencies, but leads to poor results at higher frequencies as the distances $d_{X}$ become too important in terms of wavelength. The relative difference between the edgediffracted pressures is indeed oscillating between $5 \%$ and $10 \%$, according to the analytical model in the Appendix. Poor quality results are hence obtained when the hydrophone setup does not comply with the hypothesis on the hydrophone positions being close enough to each other so that the diffracted pressure is assumed identical for the three positions. Ideally, the distance between hydrophone positions should be lower than $\lambda / 5$.

Finally, for test 3 , the distance $d_{X}=0.5 \mathrm{~cm}$ between hydrophone positions is equivalent to $\lambda / 300$ at $1 \mathrm{kHz}$ and $\lambda / 20$ at $15 \mathrm{kHz}$. For this configuration, the hypothesis is
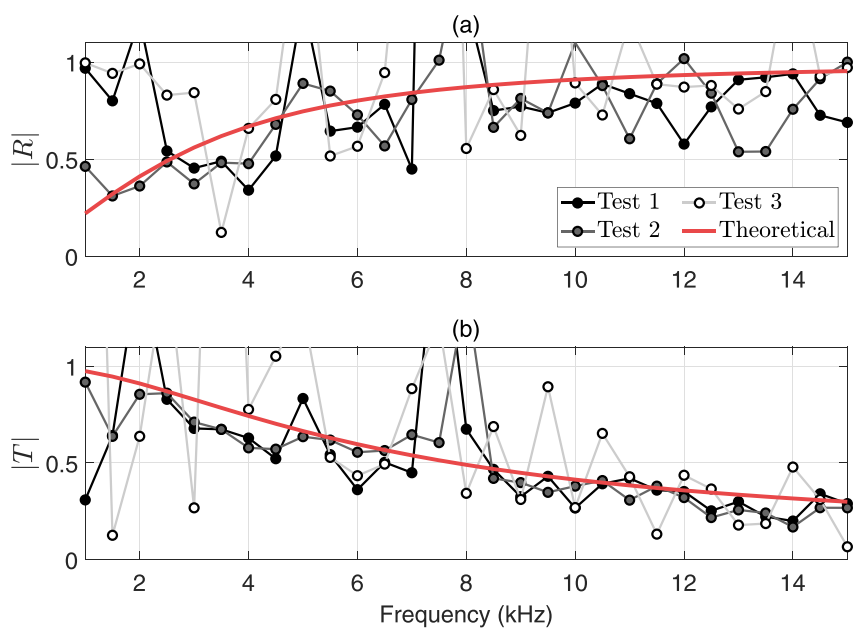

FIG. 7. (Color online) Amplitude of (a) the reflection coefficient and (b) the transmission coefficient obtained with the three-point method for the tests 1-3 defined in Table II. Reflection and transmission coefficients obtained theoretically for a panel of infinite lateral dimensions are also presented (same as in Fig. 6). satisfied and the relative difference is less than $0.1 \%$, according to the analytical model. Moreover, these three points are very close to the panel. However, the points are so close to each other that the total pressures measured are almost identical at the three points, which leads to numerical errors. Therefore, a compromise must be found to define three positions sufficiently close to each other and close to the panel while being sufficiently spaced in terms of wavelength. It is thus preferable to maintain a minimum distance of $\lambda / 20$ between two hydrophone positions.

The aforementioned criteria apply for the current aluminum panel, which is traditionally a difficult test case, and therefore will also generally apply to all panels of input impedance higher than the impedance of water. Nevertheless, it is worth noting that the behavior may be different, in terms of the edge-diffracted signal, for a panel having an input impedance lower than the impedance of water. ${ }^{9}$ For such a case, one has to make sure that measurements are made within an area where diffracted signals do exist so that it is accurately extracted from the total signal with the three-point method.

\section{PRESSURE MAPPING}

Acoustically hard panels are known for having a pronounced diffracted pattern. In order to evaluate and visualize the variations of the edge-diffracted pressure in a plane parallel to the panel, a pressure mapping is proposed. Both the analytical model in the Appendix and the three-point method are applied for a pressure mapping along the $y$ axis.

Hydrophone positions along the $x$ axis defined in Table I are considered hereafter. Along the $y$ axis, 31 positions are considered, from $y=-15 \mathrm{~cm}$ to $y=15 \mathrm{~cm}$, with $y=0 \mathrm{~cm}$ corresponding to the panel's center. The distance between each $y$-position is then $1 \mathrm{~cm}$. For frequencies $3.5 \mathrm{kHz}, 5.5 \mathrm{kHz}$, and $7.5 \mathrm{kHz}$, the calculated pressures on each side of the panel have been plotted in Fig. 8. For frequencies $14 \mathrm{kHz}, 14.5 \mathrm{kHz}$, and $15 \mathrm{kHz}$, the pressure mapping is given in Fig. 9. Finally, variations of the edge-diffracted pressure according to the model in the Appendix are visible in Fig. 10. It should be noted that Fig. 10 does not provide the amplitude of the edgediffracted pressure but only its variations.

On these pressure maps, the infinite pressure $p_{\infty}$ is almost zero everywhere, which shows that the three-point method is valid and correctly implemented. Reflected, transmitted, and incident pressures are almost identical along the $y$ axis, which reinforces the assumption of a plane wave condition. Nevertheless, on both sides of the panel, the diffracted pressure strongly varies along the $y$ axis. This last observation is consistent with the results given by the analytical model in Fig. 10. The pattern of the diffracted pressure field then depends on frequency since for low frequencies the edgediffracted pressure slowly varies along the $y$ axis, but as frequency increases, variations become steeper and closer. In addition, the center of the panel $(y=0)$ is always a maximum for the edge-diffracted pressure. Therefore, this pressure mapping highlights the strong variations of the diffracted 

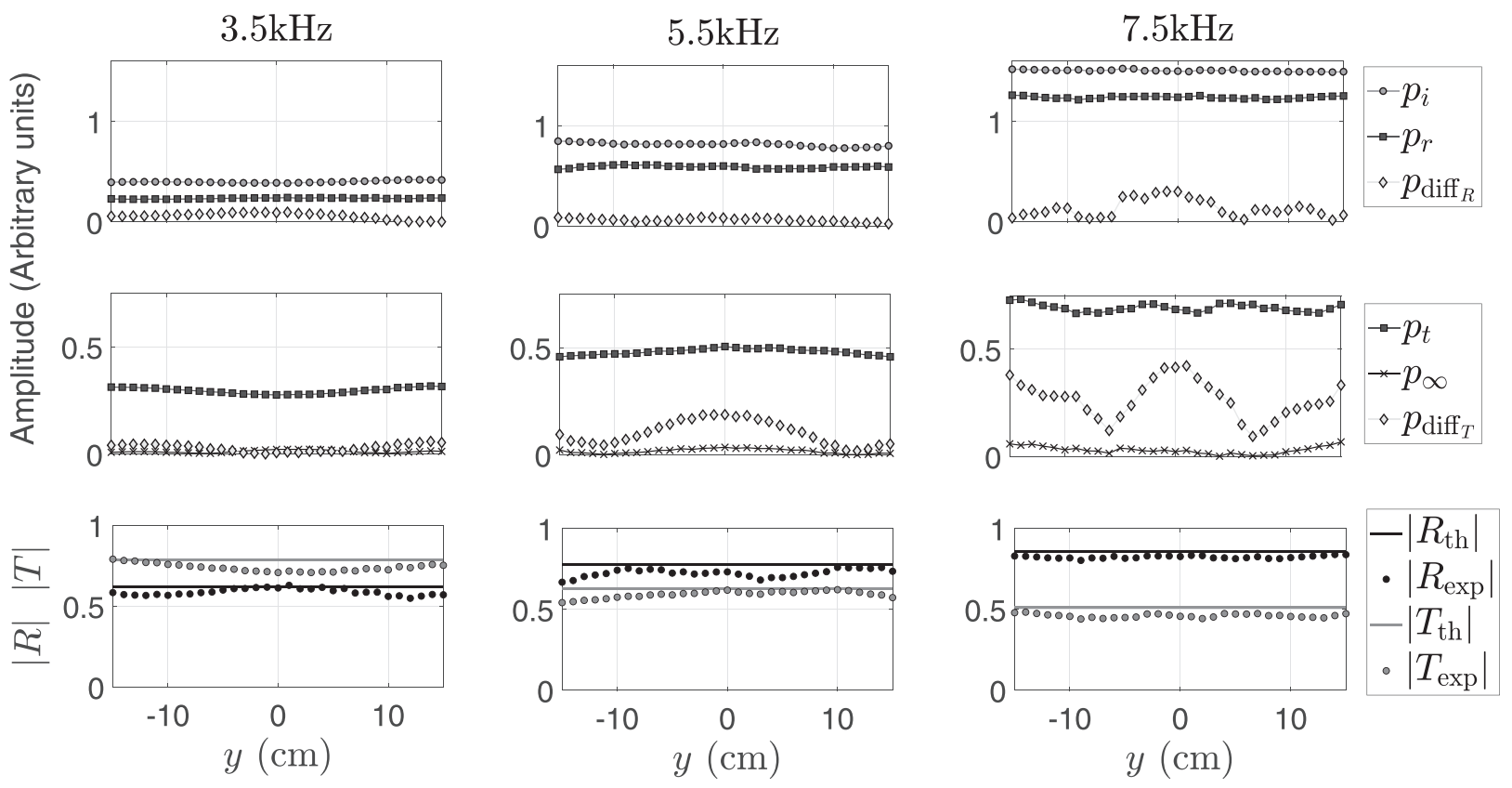

FIG. 8. Pressure mapping along the $y$ axis for $3.5 \mathrm{kHz}, 5.5 \mathrm{kHz}$, and $7.5 \mathrm{kHz}$. Experimental coefficients are also given and compared to the theoretical coefficients for a panel of infinite lateral dimensions.

pressure along the $y$ axis and shows that a mean of either reducing the edge-diffraction or calculating and removing its contribution, as the three-point method does, is crucial for accurate measurements.

\section{CONCLUSION}

Using measurements at three different positions, the three-point method yields estimations of the incident, reflected, and transmitted pressures, from which the reflection and transmission coefficients can be derived. The method also provides an estimation of the total contribution of the edgediffracted waves. The method has been applied to a homogeneous panel made of aluminum, for which the experimental reflection and transmission were in good agreement with the theoretical calculation. The validity limits of the method have then been studied. It has been shown that it is very important to choose the right positions for the hydrophones so that the assumption on the identical edge-diffracted pressure at the three points is satisfied. In Sec. IV, a mapping of the different
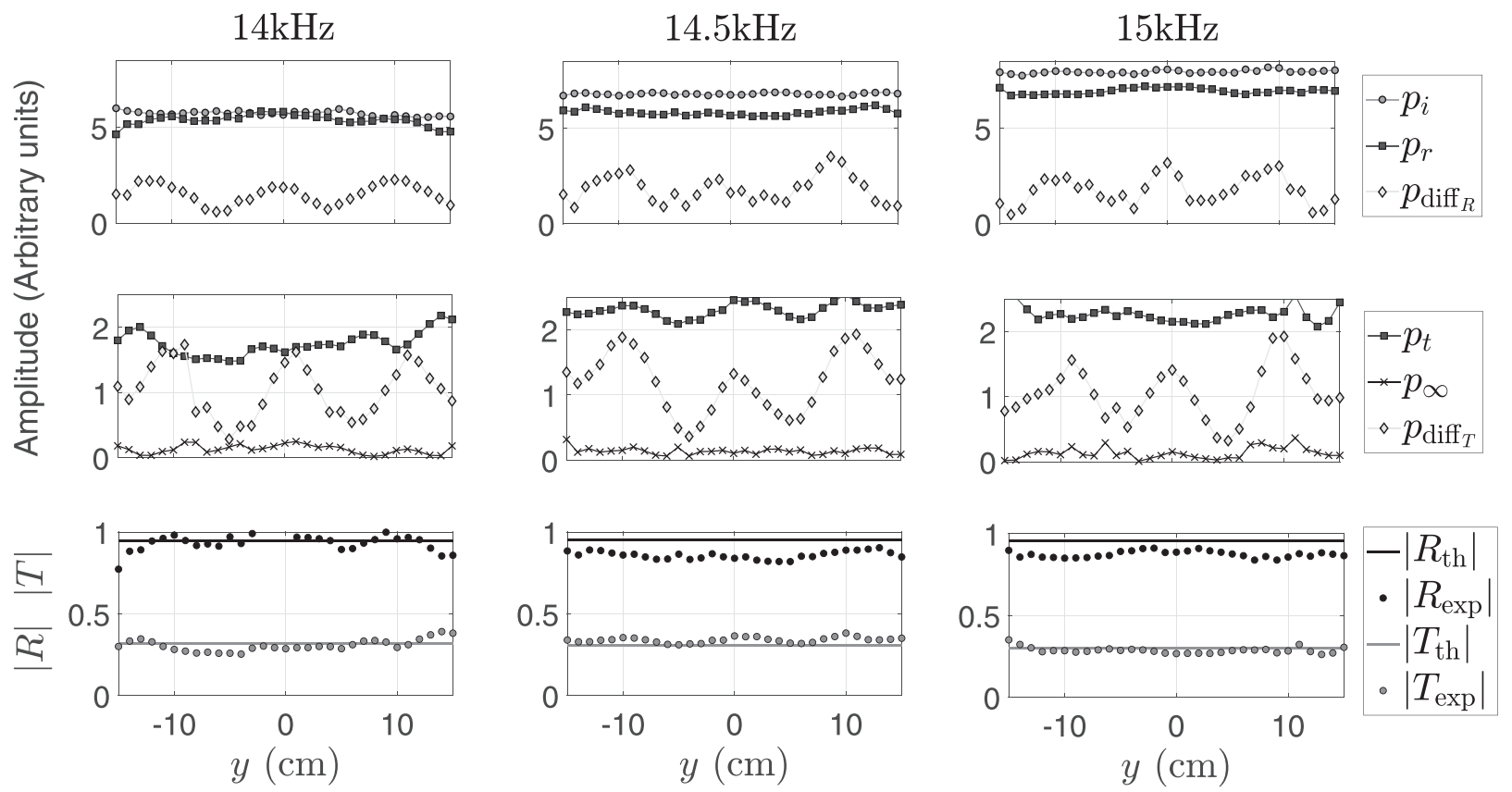

FIG. 9. Pressure mapping along the $y$ axis for $14 \mathrm{kHz}, 14.5 \mathrm{kHz}$, and $15 \mathrm{kHz}$. Experimental coefficients are also given and compared to the theoretical coefficients for a panel of infinite lateral dimensions. 

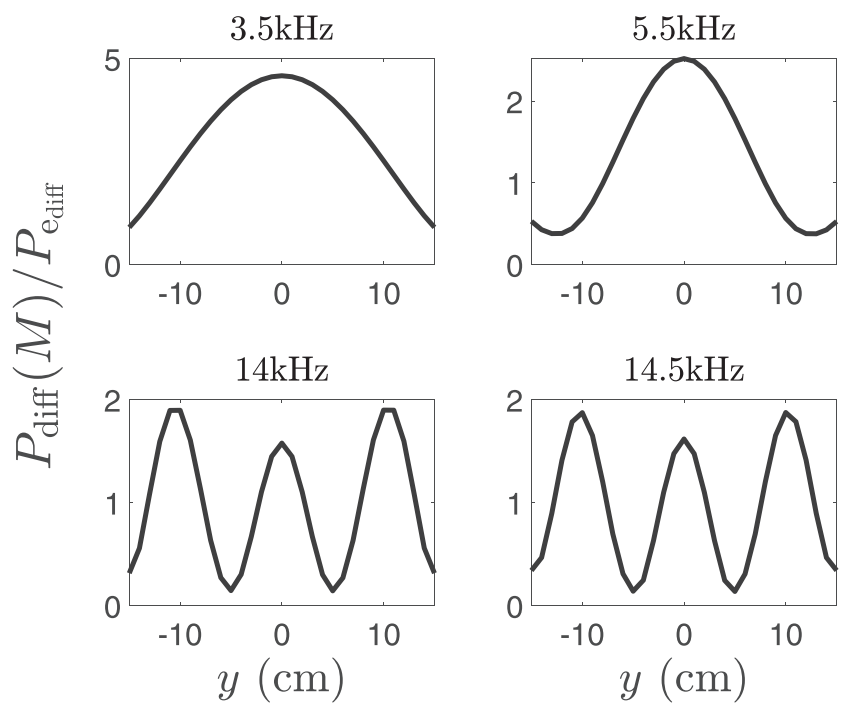

pressures along the $y$ axis was presented, where the strong variations of the edge-diffracted pressure are clearly visible.

The three-point method has the great advantage of being easy to implement, and it does not require any additional device other than the conventional experimental bench for open water tank measurements. In addition, it can be applied to acoustically hard panels, as was the case here, but also on panels made of softer materials. It can be noted that it is also possible to map the pressure fields in $(y, z)$ planes. Such mappings will be presented in future work for the case of a composite material containing resonant inclusions to test the method in the presence of complex near fields.

\section{ACKNOWLEDGMENTS}

The authors are grateful to Christian Granger, retired CNRS engineer at ISEN (Lille), for sharing his knowledge and for his help in developing the three-point method. We also thank Pierre Méresse, who, in the framework of his Ph.D., ${ }^{22}$ took part in the setting up of the experimental bench. Finally, we thank Charles Croënne for his constructive feedback.

\section{APPENDIX: EDGE-DIFFRACTED PRESSURE CONTRIBUTION}

A simple analytical model is presented here to express the diffracted pressure at one point. A square flat panel of $2 a$ side is considered and represented in Fig. 11, where the

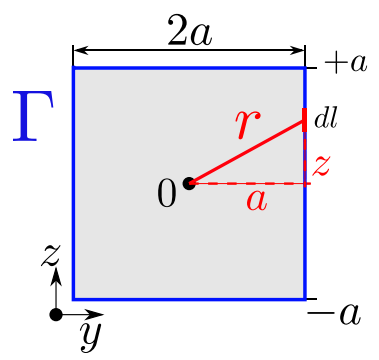

FIG. 11. (Color online) Schematic diagram of a square panel of $2 a$ side.

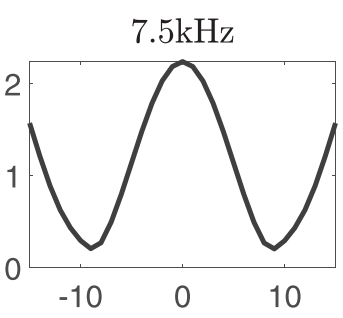

FIG. 10. Variations of the diffracted pressure along the $y$ axis obtained with

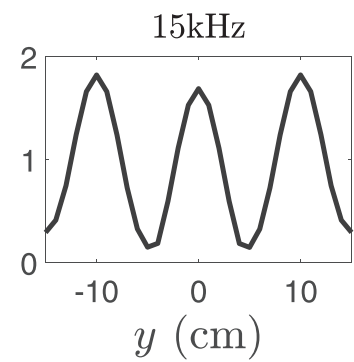
the analytical model in the Appendix.

point of coordinates $(0,0,0)$ corresponds to the center of the panel " 0. "

It is considered that each edge portion of the square behaves as a punctual source for edge-diffracted waves. It is assumed that each of these portions produces the same response to a plane wave excitation, so that the elementary pressure of edge-diffracted waves, called $P_{e_{\text {diff }}}$, is identical along edges of the square. With these simplifying considerations, the total contribution of the edgediffracted pressure at the point 0 , referred to as $P_{\text {diff }}(0)$, can be expressed using the elementary edge-diffracted pressure $P_{e_{\text {diff }}}$ and a source term that is summed on the square edges

$$
P_{\text {diff }}(0)=P_{e_{\text {diff }}} \int_{\Gamma} \frac{e^{-\mathrm{i} k r}}{r} d l
$$

where $r$ is the distance between the point 0 , where $P_{\text {diff }}$ is to be expressed, and a point on the edge. The distance $r$ is expressed as $r=\sqrt{a^{2}+y^{2}}$ for horizontal edges or $r=\sqrt{a^{2}+z^{2}}$ for vertical edges. As the panel is square, Eq. (A1) is simplified into

$$
P_{\text {diff }}(0)=4 P_{e_{\text {diff }}} \int_{-a}^{a} \frac{e^{-\mathrm{i} k \sqrt{a^{2}+l^{2}}}}{\sqrt{a^{2}+l^{2}}} d l .
$$

The edge-diffracted pressure $P_{\text {diff }}(M)$ in a point $M$ out of the panel face of coordinates $\left(x_{M}, y_{M}, z_{M}\right)$ can then be expressed using the same approach with length projections of the distance $r$ in the three-dimensional (3 D) space.

${ }^{1}$ G. Ma and P. Sheng, "Acoustic metamaterials: From local resonances to broad horizons," Sci. Adv. 2(2), 1501595 (2016).

${ }^{2}$ G. S. Sharma, A. Skvortsov, I. MacGillivray, and N. Kessissoglou, "Acoustic performance of gratings of cylindrical voids in a soft elastic medium with a steel backing," J. Acoust. Soc. Am. 141(6), 4694-4704 (2017).

${ }^{3}$ A.-C. Hladky-Hennion and J.-N. Decarpigny, "Analysis of the scattering of a plane acoustic wave by a doubly periodic structure using the finite element method: Application to Alberich anechoic coatings," J. Acoust. Soc. Am. 90(6), 3356-3367 (1991). 
${ }^{4}$ A.-C. Hladky-Hennion and J.-N. Decarpigny, "Note on the validity of using plane-wave type relations to characterize Alberich anechoic coatings," J. Acoust. Soc. Am. 92(5), 2878-2882 (1992).

${ }^{5}$ C. Audoly and C. Giangreco, "Global characterization of multilayer panels for underwater applications," J. Wave-Mater. Interact. 5(1), 55-72 (1991).

${ }^{6} \mathrm{R}$. J. Bobber, Underwater Electroacoustic Measurements (U.S. Government Printing Office, Washington, DC, 1970).

${ }^{7}$ B. Zeqiri, W. Scholl, and S. P. Robinson, "Measurement and testing of the acoustic properties of materials: A review," Metrologia 47, 156-171 (2010).

${ }^{8}$ J. C. Piquette, "Technique for detecting the presence of finite sample-size effects in transmitted wave measurements made on multilayer underwater acoustic panels," J. Acoust. Soc. Am. 90(5), 2831-2842 (1991).

${ }^{9}$ C. Audoly, "Scattering from thin elastic screens," J. Acoust. Soc. Am. 83(S1), S104 (1988).

${ }^{10} \mathrm{C}$. Audoly and C. Giangreco, "Improvement of the measurement of the transmission coefficient of panels at normal incidence using surface receivers," J. Acoust. 3(4), 369-379 (1990).

${ }^{11}$ J. D. Smith, "Diffraction from the edge of a thin elastic half plane and implications for panel measurements," J. Acoust. Soc. Am. 128(3), 973-988 (2010).

${ }^{12} \mathrm{~J}$. C. Piquette, "An analytical technique for reducing the influence of edge diffraction in reflection measurements made on thin acoustical panels," J. Acoust. Soc. Am. 80(1), 19-27 (1986).

${ }^{13}$ J. C. Piquette, "An extrapolation procedure for transient reflection measurements made on thick acoustical panels composed of lossy, dispersive materials," J. Acoust. Soc. Am. 81(5), 1246-1258 (1987).
${ }^{14}$ J. C. Piquette, "Direct measurements of edge diffraction from soft underwater acoustic panels," J. Acoust. Soc. Am. 95(6), 3090-3099 (1994).

${ }^{15}$ J. P. Szabo and A. D. Bent, "Reduction in edge effects for small panels characterized by a parametric array source," J. Acoust. Soc. Am. 145(2), 795-804 (2019).

${ }^{16} \mathrm{~V}$. F. Humphrey, "The measurement of acoustic properties of limited size panels by use of a parametric source," J. Sound Vib. 98(1), 67-81 (1985).

${ }^{17}$ P. J. Westervelt, "Parametric acoustic array," J. Acoust. Soc. Am. 35(4), 535-537 (1963)

${ }^{18}$ V. F. Humphrey, S. P. Robinson, J. D. Smith, M. J. Martin, G. A. Beamiss, G. Hayman, and N. L. Carroll, "Acoustic characterization of panel materials under simulated ocean conditions using a parametric array source," J. Acoust. Soc. Am. 124(2), 803-814 (2008).

${ }^{19}$ J. C. Piquette, "The onion method: A reflection coefficient measurement technique for thick underwater acoustic panels," J. Acoust. Soc. Am. 85(3), 1029-1040 (1989).

${ }^{20}$ J. C. Piquette, "Transmission coefficient measurement and improved sublayer material property determination for thick underwater acoustic panels: A generalization and improvement of the onion method," J. Acoust. Soc. Am. 92(1), 468-477 (1992).

${ }^{21}$ A.-C. Hennion, R. Bossut, C. Audoly, and J.-N. Decarpigny, "Analysis of the scattering of a plane acoustic wave by a periodic elastic structure using the finite element method: Application to compliant tube gratings," J. Acoust. Soc. Am. 87(5), 1861-1870 (1990).

${ }^{22} \mathrm{P}$. Méresse, "Matériaux absorbants à structure périodique et inclusions résonantes pour l'acoustique sous-marine" ("Absorbing materials with periodic arrangements and resonant inclusions for underwater acoustics"), Ph.D. thesis, University of Lille 1, 2015. 